\title{
La valeur implicite de l'homme-sievert travailleur
}

\author{
Analyse de quelques mesures adoptées dans \\ les réacteurs français à eau pressurisée *
}

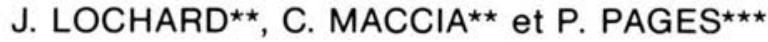 \\ (Manuscrit reçu le 8 décembre 1981)
}

\begin{abstract}
RÉSUMÉ
Cet article présente une évaluation a posteriori des valeurs implicites de l'homme-sievert résultant d'un ensemble de décisions de protection contre les expositions professionnelles dans les centrales PWR françaises. Les mesures analysées couvrent un large éventail représentatif des moyens de réduction des doses aux travailleurs : protections biologiques, modifications de matériel, mise en œuvre d'outillages spéciaux et d'automates, maquettes d'entrainement. La plupart de ces mesures sont des modifications intervenues sur les premières tranches en exploitation du programme PWR, puis intégrées au niveau de la conception des tranches ultérieures. Du point de vue méthodologique, la principale originalité réside dans la prise en compte de l'interdépendance entre les objectifs de production et de protection qui conduit à exprimer le coút final d'une mesure de protection comme le bilan des dépenses directement liées à la mesure envisagée et des coûts de production éventuellement évités.
\end{abstract}

\section{ABSTRACT}

The implicit man-sievert values resulting from a body of protection decisions concerning occupational exposures in french PWRs have been evaluated a posteriori. The measures analysed cover a wide range representative of the provisions taken to reduce occupational exposures : biological shields, modifications of equipments, utilization of special tools and robots, training mock-ups,... Most measures have been implemented during operation of the first PWRs of the French program and they have been taken into consideration at the level of the design of the later units. From the methodological point of view, the main feature is that the interdependence between production and protection aims has been taken into account. As a consequence, the final cost of a protection measure can be expressed as a balance of the expenditures directly related to the measure considered and the production costs eventually avoided.

* Cet article est la synthèse des résultats d'une étude réalisée pour le compte d'EdF-Septen.

**. CEPN (Centre d'étude sur l'évaluation de la protection dans le domaine nucléaire), BP 48, 92260 Fontenay-aux-Roses.

*** Commissariat à l'énergie atomique, IPSN, Département de protection, BP 6, 92260 Fontenay-aux-Roses. 


\section{PRÉSENTATION}

La présente étude s'inscrit dans la perspective de la gestion de la radioprotection des travailleurs dans les centrales à eau pressurisée au cours de leur fonctionnement normal. II s'agit plus particulièrement de déterminer a posteriori les valeurs implicites de l'homme-sievert résultant de certaines décisions de protection concernant les centrales PWR françaises en exploitation ou en cours de construction. L'explicitation de ces valeurs doit permettre de mieux situer l'effort déjà consenti dans le domaine de la prévention des expositions professionnelles. Par ailleurs, leur comparaison et leur analyse peut servir à terme de point de référence pour éclairer les décisions futures en matière de radioprotection des travailleurs.

II faut, cependant, noter qu'en l'état actuel de la réglementation française, il n'est fait aucune référence explicite à une quelconque valeur de l'homme-sievert en vue des choix de radioprotection et qu'en conséquence les valeurs présentées dans cet article n'ont qu'un caractère illustratif.

Le choix des options de protection analysées a été essentiellement dicté par des contraintes d'obtention des données concernant aussi bien les coûts des mesures prises que leur efficacité. Le type de quantification envisagé ici n'ayant pas fait l'objet de développements concrets dans le passé, les données directement utilisables sont rares et il convient dans la plupart des cas de rassembler des éléments épars et surtout de recourir à des estimations, voire à des hypothèses. En ce qui concerne l'évaluation des doses, le système de dosimétrie automatique en cours d'installation dans les centrales (système RAD) devrait permettre à terme une meilleure appréciation de l'efficacité des mesures envisagées. Les mesures retenues constituent, cependant, un échantillon relativement homogène quant à la qualité de l'information.

Les options analysées sont les suivantes:

01 - Dimensionnement des protections biologiques dans la travée des filtres et déminéralisateurs du bâtiment des auxiliaires nucléaires.

02 - Commande à distance des vannes des filtres et déminéralisateurs.

03 - Dimensionnement du dispositif de manutention des filtres (fig. 1).

04 - Machine de serrage-desserage des goujons de cuve (fig. 2 et 3 ).

05 - Mise en place d'un arbre intermédiaire sur les pompes primaires.

06 - Mise en place d'un monte-charge pour accéder à la piscine du bâtiment réacteur.

08 - Dispositif de manutention et de serrage des goujons des tapes trou d'homme des générateurs de vapeur (fig. 4).

09 - Dispositif de vissage des tapes d'obturation des tuyauteries primaires dans la boîte à eau des générateurs de vapeur.

10 - Maquette d'entraînement pour les interventions sur les générateurs de vapeur.

11 - Système de détection automatique des fuites des générateurs de vapeur.

12 - Machine pour le nettoyage de la bride et de la gorge de la cuve du réacteur.

13 - Machine pour le nettoyage de la bride et de la gorge du couvercle de la cuve. 
En ce qui concerne les trois premières options, plusieurs variantes ou "sous-options" ont été évaluées, car ces options générales recouvrent, en fait, des situations très disparates quant à leur efficacité.

On peut noter que la plupart des mesures sont des modifications qui sont intervenues au cours de l'exploitation des premières centrales en fonctionnement. Généralement, ces modifications ont d'abord fait l'objet d'une phase d'expérimentation sur l'un ou l'autre des réacteurs en exploitation, puis ont pu être intégrées au niveau de la conception des tranches ultérieures du programme.

Les mesures étudiées ne relèvent pas exclusivement d'un palier mais concernent l'ensemble des tranches actuellement en service (palier 900) ou en construction (palier 1300). Enfin, aucune des options étudiées ne concerne l'organisation de la radioprotection, l'efficacité de ce type de mesure étant particulièrement difficile à évaluer.

\section{LA MÉTHODE D'ÉVALUATION}

La démarche méthodologique est dans son principe très simple. Elle a fait l'objet d'une présentation détaillée dans un article précédent [1]. La valeur implicite de l'homme-sievert évité associée à une mesure technique réduisant l'exposition professionnelle n'est rien d'autre que le rapport coût de la mesure/dose collective évitée correspondante.

La dose s'évalue, généralement, à partir de débits de dose ambiants et des temps d'intervention dans le lieu ou sur le composant considéré. Quant au coût, il est défini à partir du coût d'investissement et du coût d'exploitation, les évaluations ayant été effectuées sur une base annuelle.

La principale originalité de la démarche adoptée dans cette étude réside dans la prise en compte, au niveau de l'évaluation des coûts de certaines mesures, des dépenses d'exploitation évitées qu'elles induisent.

Parmi la liste des options présentées, six d'entre elles ont une répercussion directe sur les conditions d'exploitation de la tranche. Ces options sont les suivantes:

04 - Machine de serrage-desserage des goujons de cuve (gain en disponibilité de la tranche et en main-d'œuvre) (fig. 2 et 3).

05 - Mise en place d'un arbre intermédiaire sur les pompes primaires (diminution de main-d'œuvre et gain en disponibilité).

07 - Dispositif pour la décontamination de la piscine du bâtiment réacteur (diminution de main-d'œuvre).

08 - Dispositif de manutention et de serrage des goujons des tapes trou d'homme des générateurs de vapeur (diminution de main-d'œuvre) (fig. 4).

12 - Machine pour le nettoyage de la bride et de la gorge de cuve du réacteur (gain en disponibilité et diminution de main-d'œuvre).

13 - Machine pour le nettoyage de la bride et de la gorge du couvercle de la cuve réacteur (diminution de main-d'œuvre). 
L'intégration des dimensions relevant de l'exploitation de la tranche dans les évaluations ne restitue, en fait, qu'un des objectifs possibles pouvant être associés à des mesures de protection du personnel. En effet, même si l'optimisation de la production reste l'objectif fondamental de l'exploitant et l'un des critères principaux intervenant au niveau des décisions concernant la mise en œuvre ou l'abandon des mesures visant à améliorer les niveaux d'exposition du personnel, il faut néanmoins admettre que de nombreux autres aspects rentrent également en ligne de compte. On peut citer, entre autres, les considérations relatives à la sûreté ou aux conditions de travail. Ces dimensions, bien qu'évoquées, n'ont pas été intégrées quantitativement dans la présente étude. Les problèmes méthodologiques que soulève d'ailleurs une telle perspective sont considérables et ne pourraient être résolus que par la mise en œuvre de méthodes décisionnelles beaucoup plus élaborées.

Pour l'évaluation des doses (débit de dose, temps d'intervention...) les données proviennent des mesures effectuées régulièrement dans les centrales en exploitation. En ce qui concerne les coûts et l'appréciation de l'impact des mesures sur l'exploitation des tranches (modifications du "chemin critique" (1), diminution des besoins en main d'œuvre) les données ont été recueillies auprès des différents services concernés d'Electricité de France. Par ailleurs, certaines estimations et hypothèses ont dû être retenues au lieu et place de données issues directement de l'expérience. Dans la mesure du possible, le choix des valeurs a été effectué de manière à obtenir la meilleure cohérence d’ensemble au niveau des résultats.

\section{RÉSULTATS}

\subsection{Le coût et l'efficacité des options}

Le tableau I récapitule pour l'ensemble des options et des "sousoptions" analysées, les coûts annuels de protection, les doses collectives annuelles évitées ainsi que les rapports coût/efficacité correspondants (2). Les valeurs concernant les coûts n'intègrent pas les coûts d'exploitation évités. Les rapports traduisent donc le coût implicite de l'unité de dose évitée du seul point de vue de la radioprotection. Cette démarche signifie tacitement que les coûts engagés n'ont d'autre objectif que celui de réduire l'exposition du personnel.

(1) Le "chemin critique" est défini par la succession des opérations qui déterminent le temps d'arrêt total d'un réacteur pour le renouvellement de son combustible. Les centrales PWR s'arrêtent pendant un à deux mois par an pour le renouvellement d'un tiers du combustible. Le coût d'une journée d'arrêt, c'est-à-dire le coût de remplacement de l'électricité est estimé à environ un million de francs en été et deux millions de francs en hiver pour un réacteur de $900 \mathrm{MWe}$.

(2) Pour une présentation détaillée des options et de leur évaluation, le lecteur peut se reporter à la référence [2]. 
TABLEAU I

Coûts annuels de protection, doses collectives évitées associées et rapports coût/efficacité des options analysées

\begin{tabular}{|c|c|c|c|c|}
\hline & Option & $\begin{array}{l}\begin{array}{c}\text { Coût } \\
\text { annuel }\end{array} \\
\stackrel{\Delta x}{\text { (kF/an) }}\end{array}$ & $\begin{array}{c}\text { Dose } \\
\text { collective } \\
\text { évitée } \\
\triangle S \\
\left(10^{-2} \mathrm{~h}-\mathrm{Sv}\right)\end{array}$ & $\begin{array}{c}\text { Rapport coût/ } \\
\text { efficacité } \\
\Delta_{(\mathrm{kF} / \mathrm{h}-\mathrm{S} v)}\end{array}$ \\
\hline 01 & $\begin{array}{l}\text { Protections biologiques filtres et } \\
\text { "déminés" RCV et TEP }\end{array}$ & & & \\
\hline $\begin{array}{l}\text { A } \\
B \\
D \\
E\end{array}$ & $\begin{array}{l}\text { Couloir commandes vannes, RCV } \\
\text { Couloir commandes vannes, TEP } \\
\text { Local vannes, RCV } \\
\text { Local vannes, TEP }\end{array}$ & $\begin{array}{l}0,9 \\
0,9 \\
0,45 \\
0,45\end{array}$ & $\begin{array}{l}0,17 \\
0,003 \\
0,1 \\
0,0006\end{array}$ & $\begin{array}{r}530 \\
30000 \\
450 \\
75000\end{array}$ \\
\hline $\begin{array}{c}02 \\
A \\
B\end{array}$ & $\begin{array}{l}\text { Commandes à distance } \\
\text { Local vannes, RCV } \\
\text { Local vannes, TEP }\end{array}$ & $\begin{array}{r}29,3 \\
6,1\end{array}$ & $\begin{array}{l}2,1 \\
0,2\end{array}$ & $\begin{array}{l}1400 \\
3000\end{array}$ \\
\hline $\begin{array}{c}03 \\
A \\
B\end{array}$ & $\begin{array}{l}\text { Château manutention filtres (fig. 1) } \\
\text { Passage de } 10 \text { à } 11 \mathrm{~cm} \\
\text { Passage de } 11 \text { à } 14 \mathrm{~cm}\end{array}$ & $\begin{array}{l}1,2 \\
3,6\end{array}$ & $\begin{array}{l}4,8 \\
4,3\end{array}$ & $\begin{array}{l}30 \\
80\end{array}$ \\
\hline $\begin{array}{l}04 \\
05 \\
06 \\
07 \\
08 \\
09 \\
10 \\
11 \\
12 \\
13\end{array}$ & $\begin{array}{l}\text { Machine serrage goujons cuve (fig. } 2 \text { et } 3 \text { ) } \\
\text { Arbre pompe primaire } \\
\text { Monte charge piscine BR } \\
\text { Machine décontamination piscine BR } \\
\text { Machine serrage goujons tapes GV (fig. 4) } \\
\text { Machine serrage tapes tubulures primaires } \\
\text { Maquettes GV } \\
\text { Machine détection fuites GV } \\
\text { Machine nettoyage bride et gorge cuve } \\
\text { Machine nettoyage bride et gorge couvercle }\end{array}$ & $\begin{array}{r}148 \\
306 \\
2,8 \\
78,7 \\
72 \\
23,4 \\
26,1 \\
34,5 \\
4,5 \\
4,3\end{array}$ & $\begin{array}{r}26,3 \\
2,2 \\
0,3 \\
4,8 \\
3,8 \\
3,8 \\
2,5 \\
6,4 \\
3,6 \\
2,8\end{array}$ & $\begin{array}{r}560 \\
13900 \\
870 \\
1640 \\
1900 \\
620 \\
1040 \\
540 \\
130 \\
150\end{array}$ \\
\hline
\end{tabular}

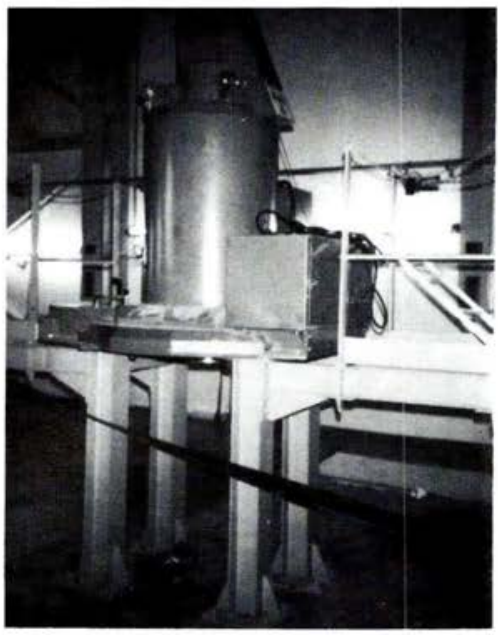

Fig. 1. - Option 03 :

château de manutention des filtres Centrale de Fessenheim.

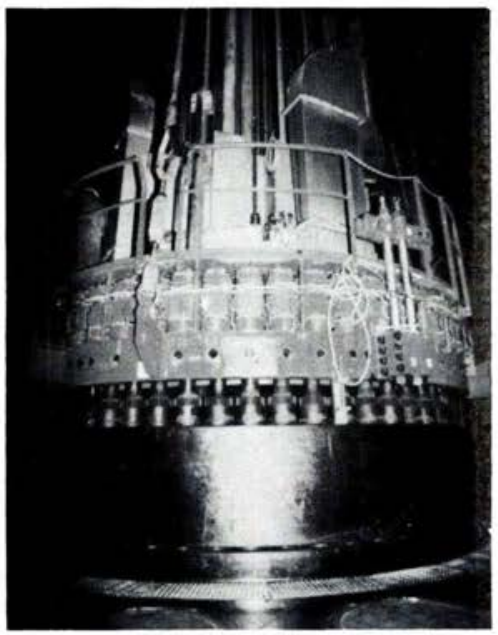

Fig. 2. - Option 04 :

machine de serrage et desserrage des goujons de cuve de réacteur utilisée respectivement sur les paliers 900 MW CP1 et CP2. 


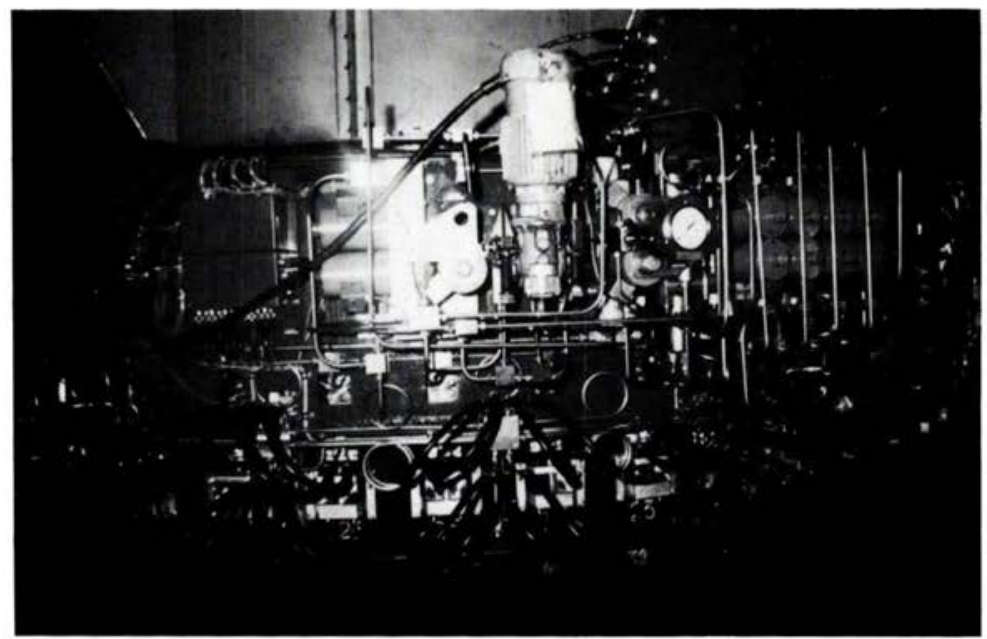

Fig. 3. - Option 04 : machine de serrage et desserrage des goujons de cuve de réacteur utilisée respectivement sur les paliers $900 \mathrm{MW}$ CP1 et CP2.

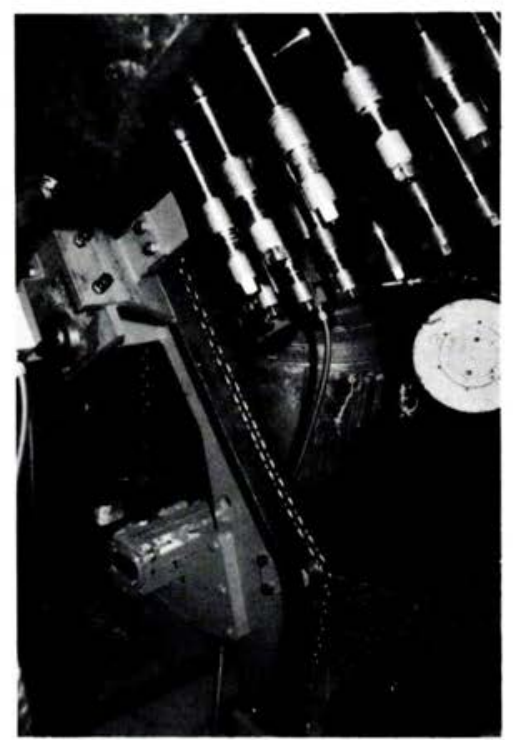

Fig. 4. - Option 08 : machine de manutention, serrage et desserrage des goujons de tape de générateurs de vapeur-paliers 900 MW CP1 et CP2. 
Le tableau II résume, pour les options qui interagissent avec les conditions de production de la tranche, les éléments de coût d'exploitation évité, soit en terme de coût de main-d'œuvre évité, soit en terme de diminution du coût de l'arrêt de la tranche. La dernière colonne du tableau présente le "bilan économique" pour chaque option.

TABLEAU ॥

Coûts d'exploitation évités et "bilan économique" des options

\begin{tabular}{|c|c|c|c|c|c|}
\hline \multirow{2}{*}{ Option } & \multirow{2}{*}{$\begin{array}{c}\text { Coût annuel } \\
\begin{array}{c}\Delta \mathrm{X} \\
\text { (kF/an) }\end{array}\end{array}$} & \multicolumn{3}{|c|}{ Coût d'exploitation évité } & \multirow{2}{*}{$\begin{array}{c}\text { Bilan } \\
\text { économique } \\
\Delta \mathrm{C}=\Delta \mathrm{X}-\Delta \mathrm{P} \\
\text { (kF/an) }\end{array}$} \\
\hline & & $\begin{array}{l}\text { Main-d'œuvre } \\
\text { (kF/an) }\end{array}$ & $\begin{array}{c}\text { Disponibilité } \\
\text { (kF/an) }\end{array}$ & $\begin{array}{c}\text { Total } \Delta \mathrm{P} \\
\text { (kF/an) }\end{array}$ & \\
\hline 04 & 148 & 21 & 4300 & 4321 & $-4173 *$ \\
\hline 05 & 306 & 2,8 & 600 & 602,8 & $-296,8^{*}$ \\
\hline 07 & 78,7 & 5 & - & 5 & 73,7 \\
\hline 08 & 72 & 5,7 & - & 5,7 & 66,3 \\
\hline 12 & 4,5 & 1,9 & 300 & 301,9 & $-297,4^{\star}$ \\
\hline 13 & 4,3 & 1,9 & - & 1,9 & 2,4 \\
\hline
\end{tabular}

Le tableau III, enfin, montre l'effet "correctif' de la prise en compte des coûts d'exploitation évités sur les rapports coût/dose évitée. Seules figurent les options qui ne conduisent pas à un "bilan" positif, c'est-à-dire à un bénéfice annuel net pour l'exploitant par rapport à la situation antérieure à la mise en œuvre de l'option.

\section{TABLEAU ॥}

\section{Variations des rapports coût-efficacité consécutifs à la prise en compte} des coûts d'exploitation évités.

\begin{tabular}{|c|c|c|}
\hline Option & $\begin{array}{c}\Delta X / \Delta S \\
(\mathrm{kF} / \mathrm{h}-\mathrm{Sv})\end{array}$ & $\begin{array}{c}\Delta \mathrm{C} / \Delta \mathrm{S} \\
(\mathrm{kF} / \mathrm{h}-\mathrm{Sv})\end{array}$ \\
\hline 04 & 5,6 & - \\
05 & 139 & - \\
07 & 16,4 & 15,3 \\
08 & 19 & 17,5 \\
12 & 1,3 & - \\
13 & 1,5 & 0,8 \\
\hline
\end{tabular}

\subsection{Le classement des options}

Le tableau IV présente le classement des options dans l'ordre croissant de leurs rapports coût-efficacité. Les deux rapports définis précédemment sont reportés et les options qui conduisent à un bénéfice net sont éliminées. 
TABLEAU IV

Classement des options

\begin{tabular}{|c|c|c|c|c|c|}
\hline \multirow[t]{2}{*}{ Option } & \multirow{2}{*}{$\begin{array}{c}\text { Coût annuel } \\
\qquad \begin{array}{c}\Delta x \\
(k F / a n)\end{array}\end{array}$} & \multirow{2}{*}{$\begin{array}{c}\text { Dose } \\
\text { collective } \\
\text { évitée } \\
\Delta S \\
\left(10^{-2} \mathrm{~h}-\mathrm{Sv} / \mathrm{an}\right)\end{array}$} & \multirow{2}{*}{$\begin{array}{c}\text { Coût } \\
\text { d'exploitation } \\
\text { évité } \\
\Delta \mathrm{P} \\
\text { (kF/an) }\end{array}$} & \multicolumn{2}{|c|}{$\begin{array}{l}\text { Rapport coût/efficacité } \\
\qquad(\mathrm{kF} / \mathrm{h}-\mathrm{Sv})\end{array}$} \\
\hline & & & & $\begin{array}{c}\Delta X / \Delta S \\
(k F / h-S v)\end{array}$ & $\begin{array}{c}(\mathrm{dX}-\Delta \mathrm{P}) / \Delta \mathrm{S} \\
(\mathrm{kF} / \mathrm{h}-\mathrm{Sv})\end{array}$ \\
\hline $03 \mathrm{~A}$ & 1,2 & 4,8 & - & 30 & 30 \\
\hline $03 \mathrm{~B}$ & 3,6 & 4,3 & - & 80 & 80 \\
\hline 13 & 4,3 & 2,8 & 1,9 & 150 & 80 \\
\hline $01 \mathrm{D}$ & 0,45 & 0,1 & - & 450 & 450 \\
\hline $01 \mathrm{~A}$ & 0,9 & 0,17 & - & 530 & 530 \\
\hline 11 & 34,5 & 6,4 & - & 540 & 540 \\
\hline 09 & 23,4 & 3,8 & - & 620 & 620 \\
\hline 06 & 2,8 & 0,3 & - & 870 & 870 \\
\hline 10 & 26,1 & 2,5 & - & 1040 & 1040 \\
\hline O2 A & 29,3 & 2,1 & - & 1400 & 1400 \\
\hline 07 & 78,7 & 4,8 & 5 & 1640 & 1530 \\
\hline 08 & 72 & 3,8 & 5,7 & 1900 & 1750 \\
\hline $02 \mathrm{~B}$ & 6,1 & 0,2 & - & 3000 & 3000 \\
\hline $01 \mathrm{~B}$ & 0,9 & 0,003 & - & 30000 & 30000 \\
\hline $01 \mathrm{E}$ & 0,45 & 0,0006 & - & 75000 & 75000 \\
\hline
\end{tabular}

\section{ANALYSE ET COMMENTAIRES}

Le premier aspect est relatif à la prise en compte des interactions entre la protection et la production. Deux constatations s'imposent. Si une mesure intervient sur le "chemin critique" des tâches de l'arrêt de tranche, elle conduit dans tous les cas à un bénéfice pour l'exploitant. Les dépenses engagées pour les options 04 (machine de serrage des goujons de cuve), 05 (arbre intermédiaire pompe primaire) et 12 (machine de nettoyage des bride et gorge de cuve) sont largement couvertes par les coûts d'exploitation évités. Ces trois exemples illustrent parfaitement comment l'optimisation de la production (c'est-à-dire ici la production d'électricité au moindre coût) n'est pas forcément contradictoire dans le cas des centrales électrogènes avec une protection renforcée des travailleurs. En ce qui concerne les gains en maind'œuvre, l'impact reste cependant assez limité surtout pour les mesures présentant un coût élevé. Dans aucun des cas analysés, la prise en compte des coûts de main-d'œuvre évités ne fait varier les rapports coût-efficacité de façon significative. Si l'on considère maintenant l'ensemble des actions de protection évaluées dans cette étude, à l'exception de l'option 04 que l'on peut admettre comme ayant été mise en œuvre pour des raisons relevant principalement de l'optimisation de l'arrêt de la tranche, le montant total des dépenses annuelles de protection s'élève à $595 \mathrm{kF}$ et le total des coûts annuels d'exploitation évités à 917 kF, soit un bénéfice d'environ $320 \mathrm{kF} / \mathrm{an}$.

Ces commentaires étant précisés, la question que l'on est en droit de se poser est celle de savoir s'il est possible, à partir des résultats de l'étude, de déterminer une valeur de l'homme-sievert qui puisse servir de référence pour éclairer les décisions concernant les mesures de protection des travailleurs envisagées dans l'avenir. 
En théorie, et si l'on accepte l'approche a posteriori, la valeur de référence devrait être le coût marginal de la dernière unité de dose évitée sous réserve que les mesures de protection aient été adoptées de façon optimale, c'est-à-dire dans l'ordre croissant de leur rapport coût/efficacité. En pratique, il est évident que les décisions interviennent au fur et à mesure de la prise en compte de l'expérience acquise et il est tout à fait possible que, parmi les décisions les plus récentes, se trouvent des options très efficaces. Finalement, la démarche la plus appropriée consiste à sélectionner parmi l'ensemble des mesures analysées, l'option ou les options les moins "coût-efficaces" indépendamment du moment de leur mise en œuvre. Si l'on se reporte au tableau IV, il s'agit des options $01 \mathrm{~B}$ et $01 \mathrm{E}$ qui conduisent à des valeurs de l'homme-sievert comprises entre 3 et 75 millions de francs. On peut noter que ces mesures sont relatives à certaines sous-options concernant le dimensionnement des protections biologiques dans la travée des filtres et déminéraliseurs. En fait, ce type de mesure recouvre des situations assez différentes quant à l'efficacité des blindages mis en œuvre puisqu'on remarque que les sous-options $01 \mathrm{D}$ et $01 \mathrm{~A}$ ont une efficacité très nettement supérieure.

Du point de vue de l'exploitant, les valeurs trouvées peuvent servir d'indicateur partiel au niveau de la pratique actuelle et future de la radioprotection dans les centrales, dans la mesure ou elles révèlent le niveau de l'effort déjà consenti. Dans cette perspective et dans un souci de cohérence interne aux décisions propres à l'exploitant, il est tout à fait possible de conserver ces valeurs comme références. Mais on peut tout aussi légitimement juger que l'effort consenti est trop important (ou trop faible) en fonction d'autres considérations et choisir, en conséquence, des valeurs différentes de l'homme-sievert.

En fait, le problème de la fixation d'une valeur de référence ne saurait trouver de solution définitive à travers la seule mise en évidence des valeurs implicites liées à la pratique de la radioprotection dans les centrales PWR françaises. Cette analyse ne constitue que la première étape qu'il faudrait poursuivre dans d'autres éléments du cycle du combustible et même dans d'autres activités industrielles.

\section{REMERCIEMENTS}

Les auteurs remercient MM. AYE et JEANSON du département

Sécurité Radioprotection Environnement d'Electricité de France pour leur précieuse collaboration à l'étude.

\section{REFERENCES}

[1] LOCHARD J., MACCIA C., PAGES P. L'évaluation de la protection radiologique des travailleurs dans les centrales nucléaires. Radioprotection, 1981, 16 201-208.

[2] LOCHARD J., MACCIA C., PAGES P. La valeur implicite de l'homme-rem travailleur. Analyse de quelques mesures adoptées dans les réacteurs français à eau pressurisée. Rapport CEPN n० 45, 1980. 